\title{
Late Thoracic Outlet Syndrome after Clavicle Fractures in Patients with Multiple Trauma: A Pitfall of Conservative Treatment
}

\author{
Daichi Ishimaru $^{1}$, Hiroyasu Ogawa ${ }^{{ }^{*}}$, Hiroshi Sumi $^{2}$, Katsuji Shimizu ${ }^{1}$ \\ ${ }^{1}$ Department of Orthopaedic Surgery, Graduate School of Medicine, Gifu University Yanagido, Gifu, Japan; ${ }^{2}$ Department of Ortho- \\ paedic Surgery, Sumi Memorial Hospital, Gifu, Japan. \\ Email: ${ }^{*}$ h-ogawa@k7.dion.ne.jp
}

Received March 23 ${ }^{\text {rd }}, 2012$; revised April 28 ${ }^{\text {th }}, 2012$; accepted May $11^{\text {th }}, 2012$

\begin{abstract}
Late thoracic outlet syndrome is a rare complication of clavicle fractures and usually warrants surgical treatment though its cause of clavicle fractures are a common injury and in most cases are treated conservatively. We report a case of a clavicle fracture accompanying severe multiple trauma, which was necessarily treated conservatively because of the severe associated injuries of the patient. The patient was unable to wear a figure-of- 8 bandage for the fracture because of the need for complete supine bed rest, due to his other injuries. In this common situation, the clavicle fracture shortened and eventually resulted in the late thoracic outlet syndrome. We believe the cause of this was because a figure-of- 8 bandage could not be applied due to the need for complete supine bed rest, and thus was inevitable because of his general condition. This case suggests that the conservative treatment of clavicle fractures, where there is the need for complete bed rest, potentially induces late thoracic outlet syndrome, and that this is indeed a pitfall in the treatment of clavicle fractures in multiple trauma.
\end{abstract}

Keywords: Clavicle Fracture; Thoracic Outlet Syndrome; Complication; Multiple Trauma

\section{Introduction}

Thoracic outlet syndrome (TOS) is a collection of upper extremity symptoms, such as pain, weakness, paresthesia, and pallor, which is caused by compression of the brachial plexus and subclavian vessels in the thoracic outlet. The cause of TOS is multifactorial, and it is frequently due to anatomical abnormalities such as hypertrophy of the scalenus muscles, cervical rib, and fibrous tissue [1]. In addition, failure of treatment of the clavicle fractures can be a rare cause [2]. In contrast to clavicle fractures, TOS as a complication is a severe condition because of the necessity of surgical treatment and possible poor outcome that includes residual upper extremity symptoms [3].

In the case of a middle part facture of the clavicle, conservative treatment using a figure-of- 8 bandage is often successfully employed [4,5]. However, the bandage cannot be correctly applied to a patient who must undertake complete bed rest for any particular reason of poor general condition. Herein, we report a case of late TOS after a clavicle fracture associated with multiple trauma, in which the cause might be due to the common situation

*Corresponding author. that patients with multiple trauma including a clavicle fracture, are necessarily treated conservatively without a figure-of- 8 bandage because of the need for complete supine bed rest. This case suggests a pitfall of the conservative treatment of clavicle fractures, resulting in the severe complication of late TOS. The patients gave the informed consent prior being included into the study.

\section{Case Report}

A 39-year-old man, who had fallen from a height of $4 \mathrm{~m}$, was referred to our hospital. He was diagnosed with multiple trauma, including a right flail chest, bilateral hemothorax, basal skull fracture, traumatic subarachnoid hemorrhage, and a right middle part clavicle fracture. Radiographs of the clavicle showed a 3-part middle part fracture of the clavicle, which was categorized into the 2B1 group by the Robinson classification (Figure 1) [6]. He had to have complete bed rest for 3 weeks for his severe other injuries.

We treated the clavicle fracture conservatively considering his general condition and the need for conservative treatment of his other injuries. After 3 weeks of complete bed rest without specific fixation of the clavicle, he im- 
proved, and he started to walk and wore a figure-of- 8 bandage for the clavicle fracture for 6 weeks. During the bed rest, the clavicle fracture was followed-up by radiography every week, and the radiograph at 3 weeks after injury showed the presence of some callus formation around the fracture site and shortening of the clavicle (Figure 2). Two months after the injury, the radiographs showed union of the clavicle, and he was able to return to his job and fully use his right arm without any symptoms such as pain or crepitation at the fracture site. However, 5 months after the injury, he began to feel numbness and imperception of the right thumb and weakened muscular strength of the right biceps muscle. Physical examination revealed hypesthesia around the right thumb, thenar eminence, and the axilla, and weakness of muscle strength in the right biceps. The Wright and Roos test for the diagnosis of TOS were positive, and other examinations, including MRI, excluded spinal or cerebral disease.

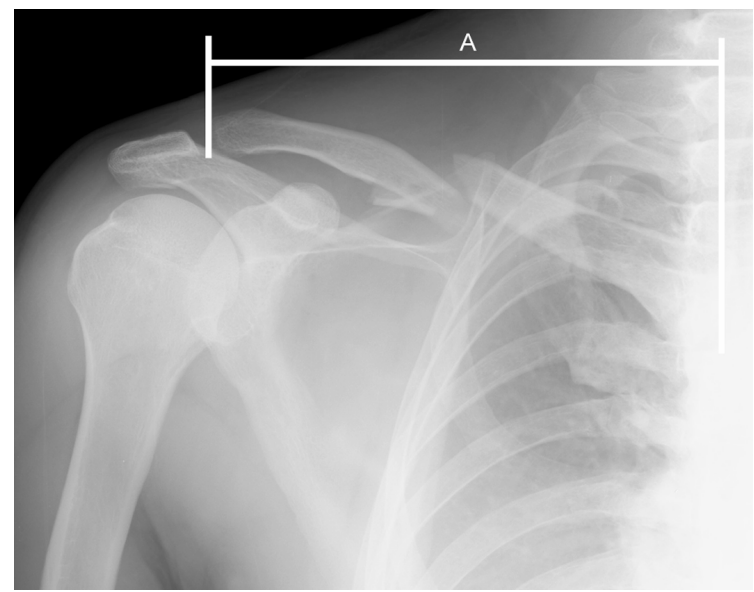

Figure 1. The radiograph of the clavicle after initial injury shows that the clavicle is divided into 3 parts, and displaced without shortening. A on the figure represents the full length of the clavicle.

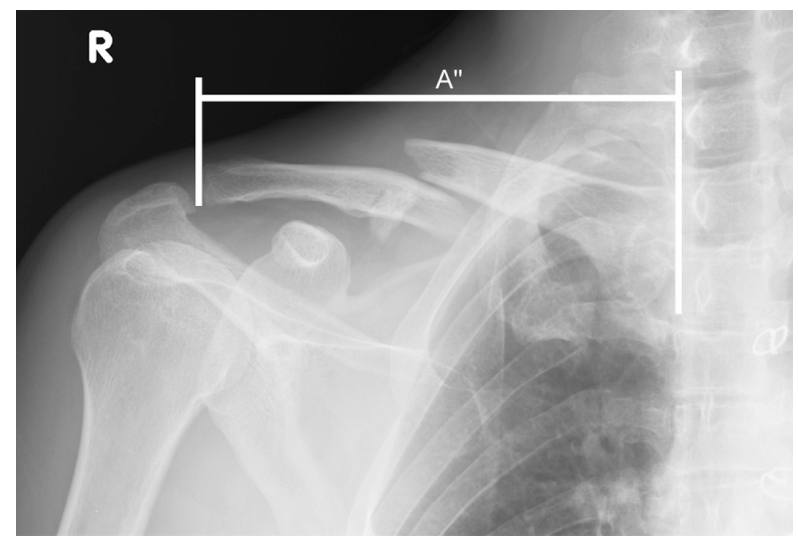

Figure 2. The radiograph of the clavicle 3 weeks after injury showing shortening between the major bony fragments of the clavicle fracture, and ratio $A " / A$ is approximately 0.8 .
The radiographic examination for the clavicle fracture showed union of the fracture. CT imaging angiography revealed that the subclavian artery and brachial plexus were compressed by callus and a distal bone fragment of the clavicle fracture (Figures 3(a) and (b)), indicating the cause of TOS. Therefore, he was diagnosed with late TOS due to the clavicle fracture, and surgery was performed.

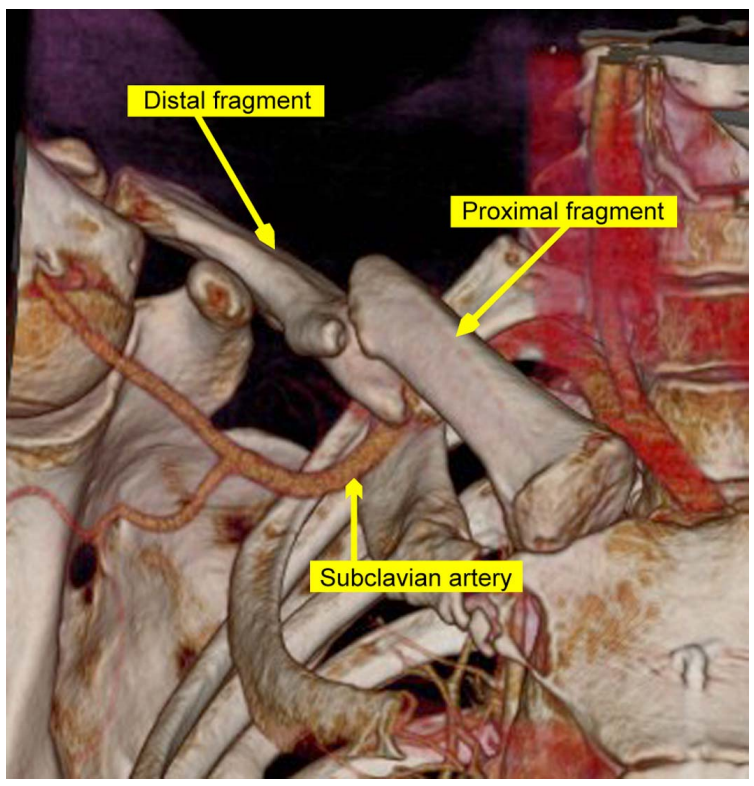

(a)

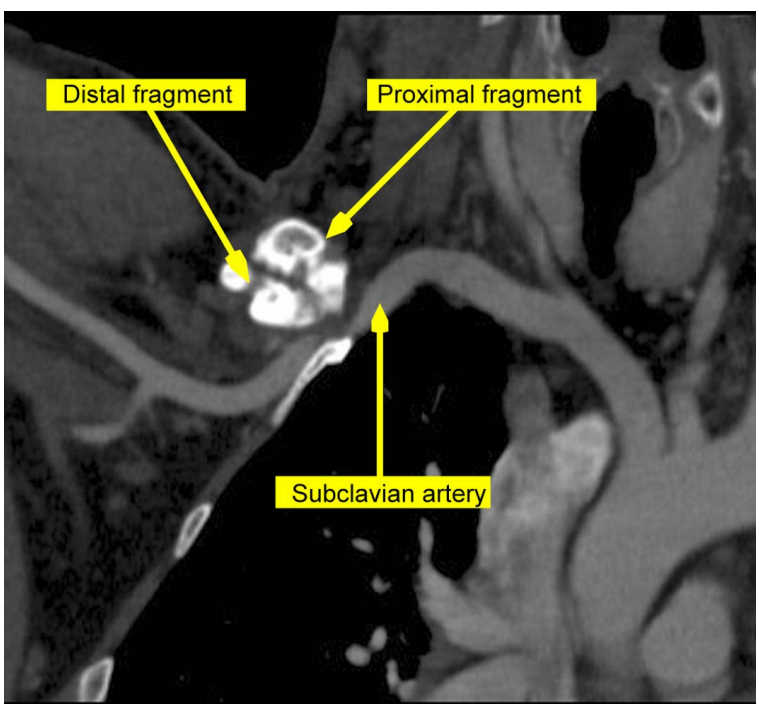

(b)

Figure 3. (a) 3-dimentional CT imaging angiography with his arm in elevated position shows that the distal fragment of the clavicle fracture compresses the brachial artery from the lateral side, and the blood flow seems to be interrupted at the fracture site; (b) CT imaging angiography reveals that the distal fragment of the clavicle protrudes toward subclavian artery, which is narrowing due to it. 


\section{Operation}

Under general anesthesia, the patient was positioned in the beach chair position, and the fracture was exposed using a supraclavicular approach. The clavicle fracture showed union including the third bony fragment in a misaligned position with callus formation. The fragment and callus were excised, and the fracture was refractured and corrected to normal alignment, and fixed with a conventional metal plate and an autologous bone graft from the ilium (Figure 4). At this time, the blood flow of the radial artery was examined with the arm in the elevated position (Wright test), but the flow was still diminished. Careful observation of the facture site revealed that not only the clavicle bone but also the soft tissue around it was shortened in the direction of the axis of the clavicle, strongly suggesting that the fibrous tissue around the fracture site had also compressed the subclavian artery and the brachial plexus. The fibrous scar around the fracture was gently detached, thereby enabling the radial blood flow to recover well, even with the arm in the elevated position.

Two weeks after the operation, the patient had obvious improvement of strength in the right biceps muscle and sensory disturbance. Eight months after the operation, the clavicle fracture united with good alignment and without excessive callus, and the symptoms of TOS completely disappeared.

\section{Discussion}

Late TOS after clavicle fractures is caused by misalignment of the fracture, excessive callus, and nonunion [2], and ironically is often more severe and troublesome than its cause. Late TOS generally requires corrective osteotomy of the clavicle, excision of excessive callus, claviculectomy, or first rib resection $[1,2,7]$, to relieve

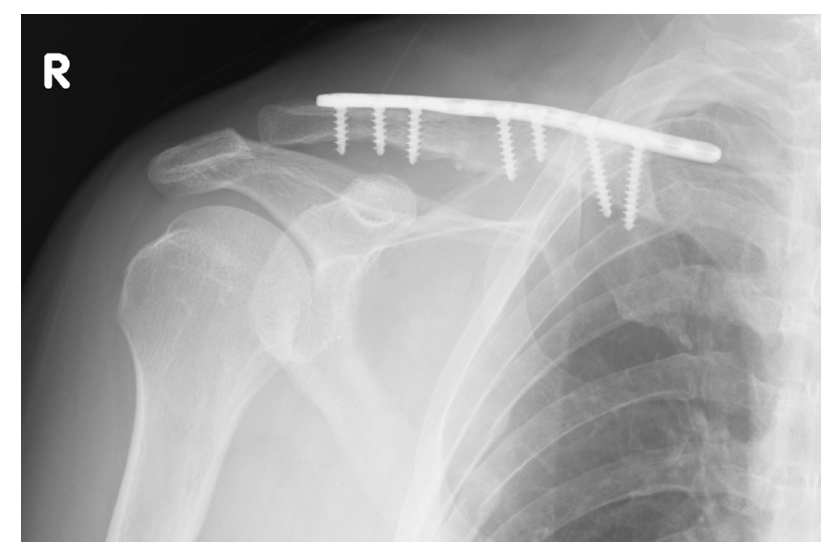

Figure 4. The radiograph of the clavicle after surgery shows that the malunion has been corrected with accurate alignment, and fixed by a conventional plate using autologous bone graft from the ilium. the symptoms, and these procedures also have a risk of recurrence of symptoms, breakage of an implant, and nonunion $[7,8]$.

Shoulder girdle injuries, including a clavicle fracture, are often caused by high energy injuries and are associated with severe multiple trauma, including head injury, great vessel injury, or thoracic injury [9]. Our patient had a clavicle fracture as a part of multiple trauma caused by a high-energy mechanism. The clavicle fracture was a common middle part fracture, accounting for approximately $80 \%$ of all clavicle fractures with generally good results by conservative treatment $[4,5,10]$. In patients with multiple trauma, including clavicle fractures, the clavicle fracture may have to be necessarily treated conservatively according to the treatment for other more severe injuries, and our patient also underwent conservative treatment for the clavicle fracture during 3 weeks of complete bed rest for other injuries. Importantly, this common situation may be a pitfall in the treatment of this fracture that can result in a complication of late TOS. The main cause might be non-wearing a figure-of- 8 bandage in supine position despite conservative treatment. Usually in middle-part fractures of the clavicle, the medial fragments are pulled cranially and dorsally by the sternocleidomastoid muscle and the lateral fragment is pulled caudally by the gravity of the upper extremity and medially by the pectoralis major and deltoid muscles, which generate displacement [11]. A figure-of-8 bandage can counteract these forces by pulling bilateral shoulder posterolaterally to correct the displacement of clavicle fractures, but this cannot be performed on patients in the supine position. Moreover, the supine position may push the shoulder anteriorly, leading to shortening of clavicle fractures in patients under complete supine bed rest. This shortening of the clavicle might be a potential contributor to late TOS [12]. In our case, the shortened clavicle during 3 weeks of complete bed rest and operative findings strongly suggest that this inevitable process in conservative treatment, which developed the shortened clavicle and soft tissue around the fracture were the main mechanisms of late TOS. This was indirectly proven by the operative finding that, in addition to the correction of the clavicle, detachment and release of the adhered soft tissues around the brachial neurovascular band was necessary for the recovery of the radial blood flow.

Our patient developed late TOS 5 months after the fracture, although the clavicle had already shortened 3 weeks after injury. Tissue fibrosis and thickening around the shortened clavicle fracture site may have gradually developed due to the influence of shoulder movement in daily life, resulting in late TOS.

In conclusion, we present a case of late TOS after a clavicle fracture. Patients with multiple trauma, which 
included clavicle fractures, often require compete bed rest in the supine position for a while for other injuries, without a figure-of- 8 bandage, and this situation might be a potential cause for the development of clavicle shortening and eventually late TOS. In the treatment of patients in such a situation, attention should be paid to the alignment of the clavicle, particularly the length, to prevent the severe complication of late TOS.

\section{REFERENCES}

[1] D. J. Chen, D. C. Chuang and F. C. Wei, "Unusual Thoracic Outlet Syndrome Secondary to Fractured Clavicle," Journal of Trauma, Vol. 52, No. 2, 2002, pp. 393-398. doi:10.1097/00005373-200202000-00033

[2] K. Fujita, K. Matsuda, Y. Sakai, H. Sakai and K. Mizuno, "Late Thoracic Outlet Syndrome Secondary to Malunion of the Fractured Clavicle: Case Report and Review of the Literature," Journal of Trauma, Vol. 50, No. 2, 2001, pp. 332-335. doi:10.1097/00005373-200102000-00022

[3] C. K. Kitsis, A. J. Marino, S. J. Krikler and R. Birch, "Late Complications Following Clavicular Fractures and Their Operative Management," Injury, Vol. 34, No. 1, 2003, pp. 69-74. doi:10.1016/S0020-1383(02)00169-9

[4] C. Faldini, M. Nanni, D. Leonetti, F. Acri, C. Galante, D. Luciani and S. Giannini, "Nonoperative Treatment of Closed Displaced Midshaft Clavicle Fractures," Journal of Orthopaedics and Traumatology, Vol. 11, No. 4, 2010, pp. 229-236. doi:10.1007/s10195-010-0113-Z

[5] A. Nordqvist, C. J. Petersson and I. Redlund-Johnell, "MidClavicle Fractures in Adults: End Result Study after Conservative Treatment," Journal of Orthopaedics and Traumatology, Vol. 12, No. 8, 2008, pp. 572-576.

\section{doi:10.1097/00005131-199811000-00008}

[6] C. M. Robinson, "Fractures of the Clavicle in the Adult. Epidemiology and Classification," The Journal of Bone \& Joint Surgery of British, Vol. 80, No. 3, 1998, pp. 476484. doi:10.1302/0301-620X.80B3.8079

[7] J. F. Connolly and M. Ganjianpour, "Thoracic Outlet Syndrome Treated by Double Osteotomy of a Clavicular Malunion: A Case Report," The Journal of Bone \& Joint Surgery of American, Vol. 84, No. 3, 2002, pp. 437-440.

[8] J. G. Skedros, B. B. Hill and T. C. Pitts, "Iatrogenic Thoracic Outlet Syndrome Caused by Revision Surgery for Multiple Subacute Fixation Failures of a Clavicle Fracture: A Case Report," Journal of Shoulder and Elbow Surgery, Vol. 19, No. 1, 2010, pp. e18-e23. doi:10.1016/i.jse.2009.05.014

[9] H. P. Gottschalk, R. H. Browne and A. J. Starr, "Shoulder Girdle: Patterns of Trauma and Associated Injuries," Journal of Orthopaedics and Traumatology, Vol. 25, No. 5, 2011, pp. 266-271. doi:10.1097/BOT.0b013e3181e47975

[10] C. Neer, "Fractures of the Clavicle," In: C. A. Rockwood Jr. and D. P. Green, Eds., Fractures in Adults, 2nd Edition, Lippincott, Philadelphia, 1984, pp. 707-713.

[11] M. Ledger, N. Leeks, T. Ackland and A. Wang, "Short Malunions of the Clavicle: An Anatomic and Functional Study," Journal of Shoulder and Elbow Surgery, Vol. 14, No. 4, 2005, pp. 349-354. doi:10.1016/j.jse.2004.09.011

[12] J. M. Hill, M. H. McGuire and L. A. Crosby, "Closed Treatment of Displaced Middle-Third Fractures of the Clavicle Gives Poor Results," The Journal of Bone \& Joint Surgery of British, Vol. 79, No. 4, 1997, pp. 537539. doi:10.1302/0301-620X.79B4.7529 